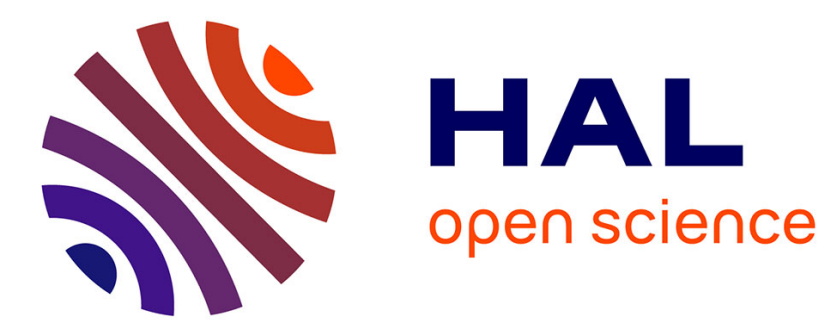

\title{
DSMC Calculations of Binary Gas Mixing in Simple Micro- Sized Configurations
}

\author{
Stavros Meskos, Stefan K Stefanov, Dimitris Valougeorgis
}

\section{To cite this version:}

Stavros Meskos, Stefan K Stefanov, Dimitris Valougeorgis. DSMC Calculations of Binary Gas Mixing in Simple Micro- Sized Configurations. AIP Conference Proceedings, 2019, Glasgow, United Kingdom. hal-02370732

\section{HAL Id: hal-02370732 \\ https://hal.science/hal-02370732}

Submitted on 20 Nov 2019

HAL is a multi-disciplinary open access archive for the deposit and dissemination of scientific research documents, whether they are published or not. The documents may come from teaching and research institutions in France or abroad, or from public or private research centers.
L'archive ouverte pluridisciplinaire HAL, est destinée au dépôt et à la diffusion de documents scientifiques de niveau recherche, publiés ou non, émanant des établissements d'enseignement et de recherche français ou étrangers, des laboratoires publics ou privés. 


\title{
DSMC Calculations of Binary Gas Mixing in Simple Micro- Sized Configurations
}

\author{
Stavros Meskos $^{1, a)}$, Stefan Stefanov ${ }^{1, b)}$ and Dimitris Valougeorgis ${ }^{2, c)}$ \\ ${ }^{1}$ Institute of Mechanics, Bulgarian Academy of Sciences - Acad. G. Bontchev St., bl. 4, 1113 Sofia, Bulgaria \\ ${ }^{2}$ Department of Mechanical Engineering, University of Thessaly - Pedion Areos, 38334 Volos, Greece \\ a)stameskos@imbm.bas.bg \\ ${ }^{b)}$ stefanov@imbm.bas.bg \\ c)diva@mie.uth.gr
}

\begin{abstract}
The Direct Simulation Monte Carlo (DSMC) method is employed to model and simulate the mixing of two non-reactive gas streams in a simple two - dimensional micro-mixer. The efficiency of the mixing process in a mixer is related to the mixing length, which is the distance from the inlet where the mixture is found to be homogeneous. A new method to measure homogeneity is presented. It has been found that the boundary conditions at the walls and the inlets, the molecular models and the physical properties of the gas species may influence the mixing process at different scales. Their level of influence on the efficiency of the mixer is presented.
\end{abstract}

\section{INTRODUCTION}

Gas mixing in micro-scale is a problem of theoretical and industrial interest for development and optimal design of gaseous MEMS/NEMS (Micro/Nano-Electro-Mechanical Systems) devices [1]. The study of the effects on the mixing process for various configurations and different boundary conditions is essential for the design of these devices. The computational analysis of gaseous flows in MEMS devices operating under different thermal conditions in non-equilibrium flow regimes cannot be based on classical continuum models of fluid motion because the continuum assumption that the flow is locally in the near-equilibrium state is no longer valid. Thus, numerical methods that solve the Boltzmann equation such as the Discrete Velocity Method and the Lattice Boltzmann Method, as well as particle based methods such as the Molecular Dynamics and the Direct Simulation Monte Carlo methods are used instead. The Direct Simulation Monte Carlo (DSMC) method [2] is arguably the most common and is employed in this work.

One of the first investigations of gas mixing in micro-configurations has been performed by Yan \& Farouk [ $\underline{3}$ ]. They investigated the dependence of the mixing length on the pressure ratio between the inlets and the outlets, between the inlets alone and on the inlet velocities. The tested configuration consisted of two parallel gas streams $\left(\mathrm{H}_{2}-\mathrm{O}_{2}\right)$ entering the mixing chamber. In the inlet region the two streams are separated by a splitter plate. It has been found that the mixing length is increased by increasing the pressure ratio between inlets and outlets and between the two inlets. In all simulations the outlet pressure is kept at $50 \mathrm{kPa}$. The same flow setup has been also examined by Wang \& $\mathrm{Li}$ [4] testing the mixing of $\mathrm{CO}$ and $\mathrm{N}_{2}$ while the outlet pressure is kept at zero (no backflow). They introduced the terms of relative density difference and mixing coefficient in order to describe better the mixing process. It has been found that the mixing length is inversely proportional to the gas temperature and the Knudsen number, while it is proportional to the Mach number. They have also shown that the wall characteristics 
have little effect on the mixing length when the rest of the properties are the same. Hassan \& Le [ $\underline{5}$ ] studied the mixing of $\mathrm{CO}$ and $\mathrm{N}_{2}$ at a T-shape micro-mixer by also keeping the outlet pressure low enough to prevent back-flow. It has been found that at higher Knudsen number the mixing length is reduced, while increasing the inlet pressure resulted in an increased mixing length. Also, increasing the flow rate and the wall temperatures reduced the mixing length, with the wall temperature effect to be more significant. Most recently, Darbandi and Sabouri [6] have investigated $\mathrm{CO}$ and $\mathrm{N}_{2}$ mixing at an angle by applying Y-shaped inlets. They have also studied the effect of replacing a "larger" micro-mixer with many "smaller" ones.

In this work, following [4], the mixing of two parallel gas streams entering the microchannel is simulated using the DSMC method. Initially, the influence of different parameters, such as temperature and accommodation coefficients at walls, inlet pressures and molecular models, on the mixing process is investigated using $\mathrm{CO}$ and $\mathrm{N}_{2}$ which have the same molecular mass. Then, in order to investigate the mixing process of gases with different molecular masses the mixing of the gas pairs of $\mathrm{He}-\mathrm{Xe}, \mathrm{Ne}-\mathrm{Ar}, \mathrm{He}-\mathrm{Ar}$ and $\mathrm{He}-\mathrm{Ne}$ is considered.

\section{PROBLEM DESCRIPTION}

The basic configuration of the micro-mixer with dimensions $\mathrm{L} / \mathrm{H}=10, \mathrm{H} / \mathrm{d}=1, \mathrm{H}=1 \mu \mathrm{m}$ is shown in Fig. 1 . The two gases enter from the left upper and lower inlets respectively. As "one" is considered the lighter species, which enters from the upper inlet, unless stated otherwise. For the case of $\mathrm{CO}$ and $\mathrm{N}_{2}$ mixing the former gas is considered as one. Pressure at each inlet is $0.2 \mathrm{~atm}$ and temperature is set at $300 \mathrm{~K}$, while vacuum is assumed at the outlet. Initially, vacuum conditions are assumed also inside the mixer. The accommodation coefficients at all walls are equal to unity, (fully diffuse reflection) and the inlet bulk velocity is zero. The area after the inlets is the "mixing chamber". The two species start mixing when they enter the mixing chamber. The mixing length is defined by the distance from the beginning of mixing chamber to a distance where the two species are found to be fully mixed.

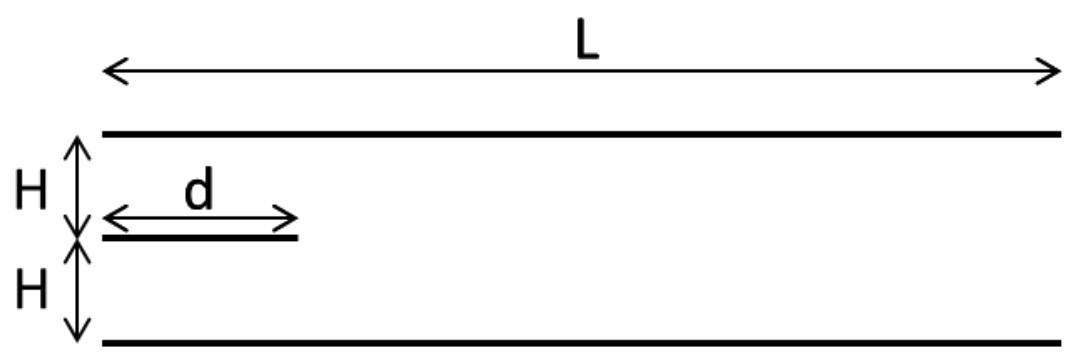

FIGURE 1. Schematic of the parallel gas micro-mixer.

The mixture is considered as fully mixed when the concentration of the species across the channel's height is equal to the ratio between the upper and lower fluxes. One way to calculate the mixing length is by measuring the homogeneity of the mixture at different lengths across the channel [4].The proposed method reads as follows:

$$
\xi_{a}^{j}=\frac{\left|n_{a}^{j, \text { up }}-n_{a}^{j, \text { low }}\right|}{\max \left(n_{a}^{j, \text { up }},_{a}^{j, \text { low }}\right)}
$$

Here, $\xi_{a}^{j}$ is the relative density difference, which will be referred to as "RDD" in this paper, of species $a$ at column $j$. Also, $n_{a}^{j, u p}$ and $n_{a}^{j, l o w}$ correspond to the number densities of the same species at the same column as measured at the two cells adjacent to the upper and lower walls of the main channel (L) respectively. Across the length of the channel it is expected that the RDD of each species will reach a minimum value. In an ideal case it would be equal to zero but for practical reasons in this work it is set at $0.5 \%$. The length at which each species is found to reach this minimum value it is the point that each species is considered to be uniformly distributed across that column. The lengths that this happens for each species might coincide or not. The larger of those two is chosen as mixing length.

Based on the same concept an alternative method is proposed in this work, where all intermediate cells are considered: 


$$
\begin{gathered}
\xi_{a}^{j}=\frac{1}{N} \sum_{i=1}^{N} \frac{\left|n_{a, i}^{j}-n_{a}^{j, a v g}\right|}{n_{a}^{j, a v g}} \\
n_{a}^{j, a v g}=\frac{1}{N} \sum_{i=1}^{N} n_{a, i}^{j}
\end{gathered}
$$

Here, $i=1, . ., N$ is the number of cells at column $j$ and $n_{a}^{j, a v g}$ is the average number density over the cells of column $j$. This approach is considered as more accurate since the average of the deviations of each cell number density of the column average is calculated.

In Fig. 2, the evolution of the RDD over the length of the mixing chamber computed with the present method and the one proposed in [4] is plotted. It is observed that the RDD evolution of both species based on the two methods is similar and this is justified by the same molecular masses of $\mathrm{CO}$ and $\mathrm{N}_{2}$.

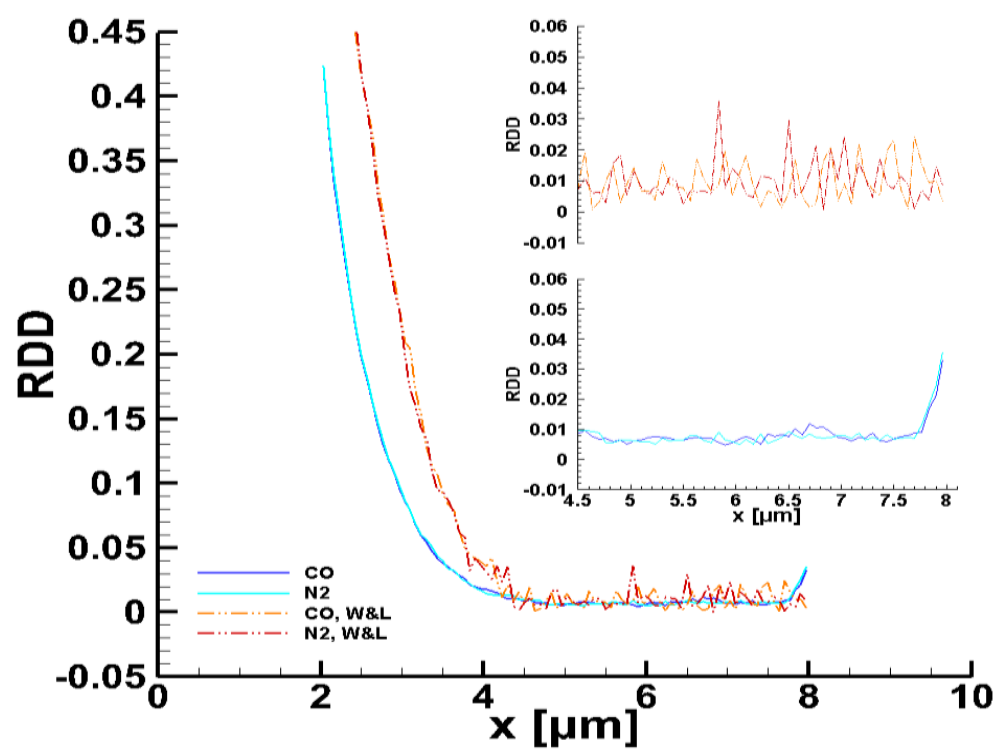

FIGURE 2. Evolution of relative density difference of $\mathrm{CO}$ and $\mathrm{N}_{2}$ calculated with both methods. At the upper right the evolutions based on the two cases are zoomed.

However, with the Wang \& Li method the statistical noise of DSMC is very significant, since the computation is based only on the extreme cells across the channel. For both cases the minimum value of RDD is about 0.005 or $0.5 \%$ at wall length equal to $4.8 \mu \mathrm{m}$ or at a mixing length equal to $2.8 \mu \mathrm{m}$. The sudden increase of the slope at the end of the RDD evolution (obtained with the proposed method) indicates enhanced separation, which is justified by the imposed vacuum at the outlet.

\section{COMPUTATIONAL CONSIDARATIONS}

The DSMC method, which is used in the present study, is a well-known particle based stochastic method that simulates gas flows very efficiently in transition and rarefied regimes. The main principle of the method is the decupling of the collisions and movement of particles. This is achieved by considering a time step smaller than the time a particle travels a mean free path at most probable velocity. Each simulated particle represents a huge amount of real molecules, usually greater than $10^{18}$. The computational domain is divided into cells. Particles are moving 
through the cells, collisions are occurring only between particles within the same cells and finally molecular speeds and number densities are sampled at each cell. This process is repeated at each time step. The simulation continues in time until a steady state is reached. There are two possibilities of averaging. The first is done by time averaging a large number of time steps after reaching the steady state. This averaging method is sufficient when only the steady state solution is of interest. However, in order to derive accurate results for the transient period, an ensemble averaging method is required as well, where many independent simulations are performed and results are additionally averaged at the same time steps by ensemble averaging over all simulations. An in-house DSMC code has been developed and employed in this work. For a rigorous mathematical description of the method, the reader is referred to [7].In Table 1, the basic DSMC configuration that has been applied in all simulations, unless stated otherwise, is presented.

TABLE 1. DSMC properties

\begin{tabular}{lc}
\hline \multicolumn{1}{c}{ Property } & Value \\
\hline Collision scheme & NTC \\
Molecular model & VSS (monoatomic) \\
Time averaging & 20 kinetic steps \\
Total samples & $500(1$ sample $=20$ kin. Steps $)$ \\
Ensemble averaging & 50 (simulations) \\
\hline
\end{tabular}

\section{RESULTS AND DISCUSSION}

The influence of the wall accommodation coefficient on the mixing length is depicted at Figures 3 (a) and (b). Figure 3(a) corresponds to the case where the accommodation coefficient is changed at all walls, while in Fig. 3(b)the accommodation coefficient is altered only at the upper wall while in all other walls is fixed to one. Results are provided with minimum relative density difference of $0.5 \%$ and $1 \%$ marked in red and blue, respectively. In Fig. 3 (a) it is seen that the mixing length is greatly reduced when moving from fully specular to fully diffuse walls. In addition, the requirement for a higher accuracy of a $0.5 \%$ results in a larger mixing length (almost up to $1 \mu \mathrm{m}$ ). This becomes more evident at Fig 3(b), where when the RDD is 1\%, the mixing does not reach the required threshold value for accommodation coefficient values in the range below 0.3 , while when the RDD is $0.5 \%$ this is happening in a much wider range of values less than 0.7. It is evident that the wall material and the type of gas-surface interactions have a significant influence on the mixing process.

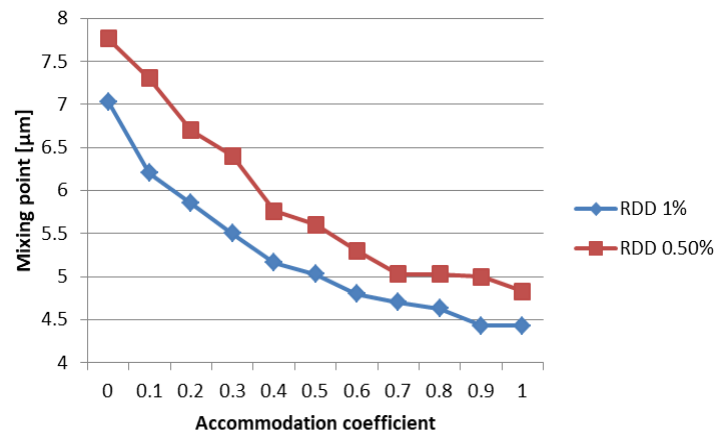

(a)

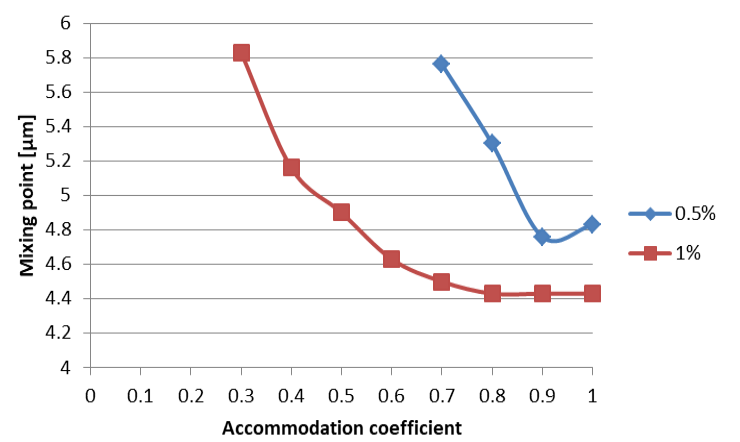

(b)

FIGURE 3. Mixing length variation over different accommodation coefficients $\alpha$ at walls: (a) all walls have the same $\alpha \in[0,1]$ and (b) the upper wall has $\alpha \in[0,1]$, while all other walls have $\alpha=1$. 
Table 2 compares the mixing length for different temperatures at walls, while the temperature at the inlets is fixed at $300 \mathrm{~K}$. While the temperature is increased at all walls at 1.5 and 2 times, from $300 \mathrm{~K}$ to $450 \mathrm{~K}$ and $600 \mathrm{~K}$ respectively, only a very small decrease in the mixing length is observed. On the third column the optimum RDD achieved at this length is shown and its analysis suggests that although the length is reduced the relative density difference is increased which makes the mixture less homogenous. This remark is further strengthened by the average RDD at the end of the mixing chamber tabulated in the last column of Table 1. As the wall temperature is increased the separation effect of the mixture becomes stronger.

TABLE 2. Influence of the wall temperature on the mixing length.

\begin{tabular}{cccc}
\hline Temperature [K] & $\begin{array}{c}\text { Mixing length } \\
{[\boldsymbol{\mu m}]}\end{array}$ & RDD & Average RDD \\
\hline 300 & 2.8 & $0.57 \%$ & $0.76 \%$ \\
450 & 2.7 & $0.67 \%$ & $0.98 \%$ \\
600 & 2.5 & $0.69 \%$ & $1 \%$ \\
\hline
\end{tabular}

All simulations have been performed by employing the Variable Soft Sphere (VSS) molecular model, proposed by Koura and Matsumoto [8]. In addition, the Variable Hard Sphere (VHS) and the Hard Sphere (HS) models, proposed by Bird [9] have also been investigated and the results have been compared with the ones obtained by the VSS model. The readers are referred to [10] where a detailed and intuitive description of the phenomenological models is provided. Table 3 depicts the mixing length variations over the different models.

TABLE 3. Influence of the molecular model on the mixing length.

\begin{tabular}{cccc}
\hline \multirow{2}{*}{$\begin{array}{c}\text { Molecular } \\
\text { Model }\end{array}$} & $\begin{array}{c}\text { Mixing length } \\
{[\boldsymbol{\mu \mathrm { m } ]}}\end{array}$ & \multicolumn{2}{c}{ Relative Error } \\
\cline { 3 - 4 } & & VSS/VHS & VSS/HS \\
\hline HS & 3.3 & & \\
VHS & 3.15 & $12.4 \%$ & $17.4 \%$ \\
VSS & 2.8 & & \\
\hline
\end{tabular}

It is interesting that even for the flow setups with isothermal walls there is a significant difference between the models. In order to better understand the origin of this difference a comparison of the corresponding velocity profiles is conducted. The velocity profiles of the mixture for the VSS case are plotted at the beginning, the middle and the end of the mixing chamber in Fig. 4 as the reference case. Then, a comparison is done at three different points on the center line at each length for each model respectively and Table 4 tabulates the differences in the velocities.

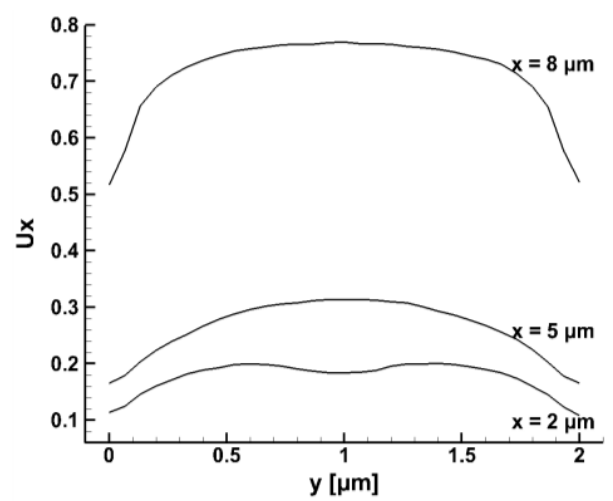

FIGURE 4. Velocity profiles of the mixture, for the VSS case, at the start, middle and end of the mixing chamber respectively. Velocity reference is the most probable velocity of CO at $300 \mathrm{~K}(422.08 \mathrm{~m} / \mathrm{s})$. 
TABLE 4. Comparison of velocities for different physical models.

\begin{tabular}{ccccccc}
\hline \multirow{2}{*}{$\begin{array}{c}\text { Length in } \\
\text { Mixing } \\
\text { chamber } \\
{[\boldsymbol{\mu \mathrm { m } ]}]}\end{array}$} & \multicolumn{3}{c}{ Velocity $(\times \mathbf{4 2 2 . 0 8} \mathbf{~ m / s})$} & \multicolumn{3}{c}{ Relative Error } \\
\cline { 2 - 7 } & VSS & VHS & HS & VSS/VHS & VSS/HS & VHS/HS \\
\hline 2 & 0.1837 & 0.1885 & 0.1858 & $2.61 \%$ & $1.14 \%$ & $1.43 \%$ \\
5 & 0.3133 & 0.3232 & 0.3172 & $3.16 \%$ & $1.24 \%$ & $1.88 \%$ \\
8 & 0.7692 & 0.7785 & 0.7682 & $1.21 \%$ & $0.13 \%$ & $1.32 \%$ \\
\hline
\end{tabular}

Although the differences are relatively small, the influence on the mixing process is large, which means that the velocity of the flow field is also a significant parameter. The authors in $[3,4]$ arrived in the same conclusion by investigating different inlet bulk velocities. This result supports the Koura and Matsumoto [] conclusion that the VSS should be preferred in cases of gas mixture flows as a more reliable model.

The effect of the molecular mass difference has also been investigated by considering species with different molecular masses. In Table 5 the results of mixing lengths and RDDs are provided for the following combinations: $\mathrm{He}-\mathrm{Xe}, \mathrm{Ne}-\mathrm{Ar}, \mathrm{He}-\mathrm{Ar}$ and He-Ne. Compared to the base case where the two species with similar masses are mixed, now the relative density difference evolves differently.

TABLE 5. Mixing lengths and RDDs for species with different physical properties

\begin{tabular}{cccccccc}
\hline Species & RDD1 & $\begin{array}{c}\text { Point 1 } \\
{[\boldsymbol{\mu} \mathbf{m}]}\end{array}$ & RDD2 & $\begin{array}{c}\text { Point 1 } \\
{[\boldsymbol{\mu \mathrm { m }}]}\end{array}$ & $\begin{array}{c}\text { Mixing length } \\
{[\boldsymbol{\mu m}]}\end{array}$ & $\mathbf{m 1} / \mathbf{m} 2$ & $\mathbf{d 1} / \mathbf{d 2}$ \\
\hline $\mathrm{He}-\mathrm{Xe}$ & $0.6 \%-1 \%$ & 4.23 & $1.4 \%-2.4 \%$ & 4.16 & 2.23 & 0.03 & 0.404 \\
$\mathrm{Ne}-\mathrm{Ar}$ & $0.7 \%-1.1 \%$ & 4.23 & $1 \%$ & 4.16 & 2.23 & 0.506 & 0.659 \\
$\mathrm{He}-\mathrm{Ar}$ & $0.5 \%-1.1 \%$ & 4.3 & $1.1 \%-1.6 \%$ & 2.56 & 2.56 & 0.1 & 0.559 \\
$\mathrm{He}-\mathrm{Ne}$ & $0.7 \%-1 \%$ & 4.3 & $1 \%-1.5 \%$ & 2.5 & 2.5 & 0.2 & 0.845 \\
\hline
\end{tabular}

Several interesting phenomena take place. The lighter species can achieve a much better uniform distribution having RDDs $0.5 \%-0.7 \%$ at a length of about $4.3 \mu \mathrm{m}$, while the heavier species never reach that good distribution, with a minimum RDD of about $1 \%$ and moving up to $2.4 \%$. In addition, the lighter species is always influenced by the heavier one loosing very quickly its uniformity. Furthermore, the final mixing length is similar in all the cases. The main difference and influence of the heavier species lies on the RDD. This means that different cases may result in the same mixing lengths but with different homogeneity at those corresponding lengths. It is important to note that these species will never be "fully mixed" and only a best possible mixing can be achieved, depending on the boundary conditions. This can be better understood from Fig. 5, where the evolution of RDD for the case of He-Xe, are given with colors (red for $\mathrm{He}$ and blue for $\mathrm{Xe}$ ). The two species are distributed at different rate and their RDD evolution lines never cross or coincide. In addition, at the same figure the RDD evolution of He-Xe mixture is plotted with green for He and black for Xe in a mixing chamber where its length has been increased by $4 \mu \mathrm{m}$ (total length of the mixer becomes $12 \mu \mathrm{m}$ ). This is performed in order to investigate the influence of the length on the final results. The behavior is identical. In both cases both species reach the minimum value of RDD at the same point and after that they expand towards vacuum in a similar manner. 


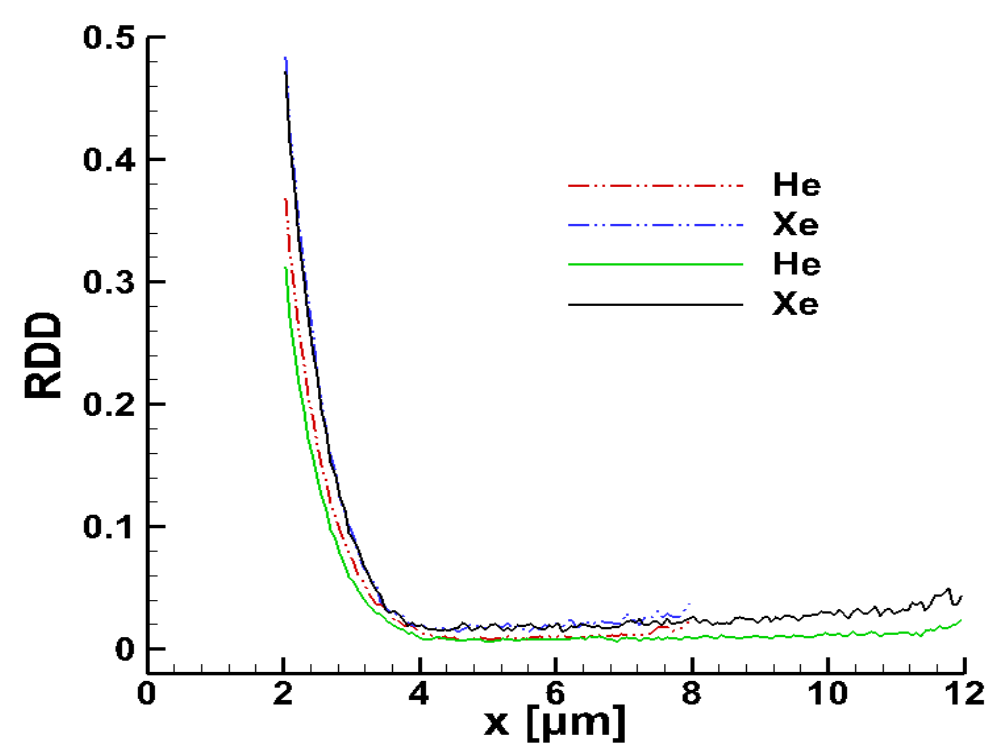

FIGURE 5. Evolution of relative density difference for the case of He-Xe for mixing chambers with total length of $8 \mu \mathrm{m}$ (red and blue) and $12 \mu \mathrm{m}$ (green and black).

\section{CONCLUSIONS}

The mixing process of two parallel gas streams of monoatomic, non-reactive species have been investigated in terms of the mixing length and homogeneity of the mixture. The boundary conditions at the walls and the inlets of the mixer, the molecular model and the molecular masses of the species have been shown to have different degree of influence to the mixing process. In order to describe correctly that process and compare the efficiency of different micro-mixers it is necessary to define along with the mixing length the corresponding measure of homogeneity. In this work a new type of measurement is proposed by evaluating how uniformly each species is distributed in space. By accepting a very small value, usually about $0.5 \%$ the condition of "fully mixed" flow practically is considered as fulfilled. Even a small change in that value can lead to errors. More predominantly for the realistic case of mixing species with different masses this value does not stay fixed; rather it varies differently for each species, giving as well a good indication of the separation effects that take place. Moving from specular to diffuse walls the mixing process is greatly improved. Increasing the wall temperature, with fixed inlet gas temperature, results in a small decrease of the mixing length on the account of the homogeneity. Simulations performed using three different intermolecular models of VSS, VHS and HS resulted in differences in the mixing length that should not be neglected. An explanation for that is related to the observation that the application of different molecular interaction models reflects in small differences in velocity fields of the gas components. Finally, the difference between molecular masses of the two species influences the mixing process mainly on the mixture homogeneity, while the mixing length remains almost unaffected.

\section{ACKNOWLEDGMENTS}

This project has received funding from the European Union's Framework Programme for Research and Innovation Horizon 2020 (2014-2020) under the Marie Sklodowska-Curie Grant Agreement No. 643095.

\section{REFERENCES}

1. $\quad$ C.-Y. Lee, C.-L. Chang, Y.-N. Wang, and L.-M. Fu Int. J. Mol. Sci.12(5), 3263-3287 (2011).

2. G. A. Bird, Molecular Gas Dynamics and the Direct Simulation of Gas Flows (Clarendon Press, Oxford, 1994)

3. F. Yan and B. Farouk,J. Microscale Thermophysical Eng.6:3, 235-251 (2002).

4. M. Wang and Z. Li,Int. J. of Heat \& Mass Transfer49, 1696-1702 (2006)

5. M. Le and L. Hassan, Appl. Therm. Eng.27, 2370-2377 (2007) 
6. M. Darbandi and M. Sabouri, Sensors and Actuators B: Chemical31, 78-83 (2015)

7. S. Stefanov, SIAM J. Sci. Comput., 33(2), 677-702 (2011).

8. K. Koura and H. Matsumoto, Phys. Fluids A3, 2459 (1991)

9. G. A. Bird, "MONTE-CARLO SIMULATION IN AN ENGINEERING CONTEXT" in $12^{\text {th }}$ Rarefied Gas Dynamics Symposium-1980,Progress in Astronautics and Aeronautics, Rarefied Gas Dynamics, Parts I and II,1981, pp. 239-255.

10. G. A. Bird The DSMC Method Version 1.2 (Amazon Distribution GmbH, Leipzig, 2013) pp. 49-54 